\title{
Correction to: Acoustic black holes: massless scalar field analytic solutions and analogue Hawking radiation
}

\author{
H. S. Vieira ${ }^{1,2}$ • V. B. Bezerra ${ }^{1}$
}

Received: 8 February 2019 / Accepted: 11 March 2019 / Published online: 1 April 2019

(c) Springer Science+Business Media, LLC, part of Springer Nature 2019

\section{Correction to: Gen Relativ Gravit (2016) 48:88 https://doi.org/10.1007/s10714-016-2082-x}

In Sect. 2, there is a mistake when we transformed Eq. (16) into Eq. (21), by using the new coordinate given by Eq. (17) and its consequences expressed in Eqs. (18)-(20). Correcting this mistake, the equation corresponding to Eq. (21) should read

$$
\begin{aligned}
& \frac{d^{2} R}{d x^{2}}+\left(\frac{1}{x-x_{h}}-\frac{1}{2 x}\right) \frac{d R}{d x} \\
& +\left[\frac{m^{2}\left(B^{2}+c^{2} x_{h}\right)}{4 c^{2} x_{h}^{2}} \frac{1}{x}+\frac{-B^{2} m^{2}-c^{2} m^{2} x_{h}+c^{2} x_{h}^{2} \omega^{2}}{4 c^{2} x_{h}^{2}} \frac{1}{x-x_{h}}\right. \\
& \left.+\frac{\left(c x_{h} \omega-B m\right)^{2}}{4 c^{2} x_{h}} \frac{1}{\left(x-x_{h}\right)^{2}}\right] R=0,
\end{aligned}
$$

where we used another relation for the new radial coordinate instead of Eq. (17), given by

$$
x=r^{2}
$$

The original article can be found online at https://doi.org/10.1007/s10714-016-2082-x.

$凶$ H. S. Vieira

horacio.santana.vieira@hotmail.com

V. B. Bezerra

valdir@fisica.ufpb.br

1 Departamento de Física, Universidade Federal da Paraíba, Caixa Postal 5008, João Pessoa, PB CEP 58051-970, Brazil

2 Centro de Ciências, Tecnologia e Saúde, Universidade Estadual da Paraíba, Araruna, PB CEP 58233-000, Brazil 
in such a way to obtain an equation which is identified with a confluent Heun equation. Now, using this new coordinate, Eq. (18) is written as

$$
\Delta=x-\frac{A^{2}}{c^{2}} .
$$

Thus, the new horizon surface equation of the rotating acoustic black hole given by Eq. (19) should read

$$
\Delta=x-x_{h}=0,
$$

and Eq. (20) turns into

$$
x_{h}=r_{h}^{2}=\frac{A^{2}}{c^{2}}
$$

which corresponds to the new event horizon of the rotating acoustic black hole. In what follows, let us do homographic substitution given by Eq. (22). Thus, Eq. (23) should read

$$
\begin{aligned}
& \frac{d^{2} R}{d z^{2}}+\left(\frac{1}{z}+\frac{-1 / 2}{z-1}\right) \frac{d R}{d z} \\
& +\left\{\frac{B^{2} m^{2}+c^{2} m^{2} x_{h}-c^{2} x_{h}^{2} \omega^{2}}{4 c^{2} x_{h}} \frac{1}{z}+\frac{-m^{2}\left(B^{2}+c^{2} x_{h}\right)}{4 c^{2} x_{h}} \frac{1}{z-1}\right. \\
& \left.\quad-\left[i\left(\frac{c x_{h} \omega-B m}{2 c \sqrt{x_{h}}}\right)\right]^{2} \frac{1}{z^{2}}\right\} R=0 .
\end{aligned}
$$

The F-homotopic transformation of the dependent variable, $R(z) \mapsto U(z)$, is such that

$$
R(z)=z^{A_{1}} U(z)
$$

where the coefficient $A_{1}$ given by Eq. (25) is now expressed as

$$
A_{1}=i\left(\frac{c x_{h} \omega-B m}{2 c \sqrt{x_{h}}}\right) \text {. }
$$

In this case, Eq. (26) should be changed to

$$
\begin{aligned}
& \frac{d^{2} U}{d z^{2}}+\left(\frac{2 A_{1}+1}{z}+\frac{-1 / 2}{z-1}\right) \frac{d R}{d z} \\
& +\left[\frac{B^{2} m^{2}-i B c m \sqrt{h_{h}}+c^{2} m^{2} x_{h}+i c^{2} x_{h}^{3 / 2} \omega-c^{2} x_{h}^{2} \omega^{2}}{4 c^{2} x_{h}} \frac{1}{z}\right. \\
& \left.-\frac{B^{2} m^{2}-i B c m \sqrt{h_{h}}+c^{2} x_{h}\left(m^{2}+i \sqrt{x_{h}} \omega\right)}{4 c^{2} x_{h}} \frac{1}{z-1}\right] R=0 .
\end{aligned}
$$


This functional form of the general solution of the radial part of the Klein-Gordon equation for a massless scalar field in the spacetime of a rotating acoustic black hole, in the exterior region of the acoustic event horizon, is similar to Eq. (30), and therefore, its solution can be written in terms of the confluent Heun function as

$$
\begin{aligned}
R(z)= & z^{\frac{\beta}{2}}\left\{C_{1} \operatorname{HeunC}(\alpha, \beta, \gamma, \delta, \eta ; z)\right. \\
& \left.+C_{2} z^{-\beta} \operatorname{HeunC}(\alpha,-\beta, \gamma, \delta, \eta ; z)\right\},
\end{aligned}
$$

where $C_{1}$ and $C_{2}$ are constants, and the parameters $\alpha, \beta, \gamma, \delta$, and $\eta$, which are given originally by Eqs. (31)-(35), are now given by the following expressions

$$
\begin{aligned}
\alpha & =0 \\
\beta & =\frac{i\left(c x_{h} \omega-B m\right)}{c \sqrt{x_{h}}} ; \\
\gamma & =-\frac{3}{2} \\
\delta & =-\frac{x_{h} \omega^{2}}{4} ; \\
\eta & =\frac{1}{4}\left[3-m^{2}\left(1+\frac{B^{2}}{c^{2} x_{h}}\right)+x_{h} \omega^{2}\right] .
\end{aligned}
$$

As a conclusion, the solution of the radial part of the Klein-Gordon equation is formally the same as Eq. (30). In fact $\alpha$ assumes the same value and $\delta$ is given, formally, by the same expression as shown in Eq. (34). As a consequence of the fact that the solution is the same, the analogue Hawking radiation given by Eqs. (37)-(60) is all correct and therefore should be preserved.

In Sect. 3, a similar mistake was done when we transformed Eq. (71) into Eq. (78), using the coordinate transformation given by Eq. (72). Now, let us correct this mistake. In order to do this, let us write the line element which describes a canonical acoustic black hole in a more appropriate form given by

$$
d s^{2}=-c^{2} \Delta d \tau^{2}+\Delta^{-1} d r^{2}+r^{2}\left(d \theta^{2}+\sin ^{2} \theta d \phi^{2}\right)
$$

where in this case Eq. (65) should read

$$
\Delta=1-\frac{r_{0}^{4}}{r^{4}}
$$

The covariant Klein-Gordon equation in the spacetime of a canonical acoustic black hole, given by Eq. (69), is now written as

$$
\left[-\frac{r^{2}}{c^{2} \Delta} \frac{\partial^{2}}{\partial \tau^{2}}+\frac{\partial}{\partial r}\left(r^{2} \Delta \frac{\partial}{\partial r}\right)+\frac{1}{\sin \theta} \frac{\partial}{\partial \theta}\left(\sin \theta \frac{\partial}{\partial \theta}\right)+\frac{1}{\sin ^{2} \theta} \frac{\partial^{2}}{\partial \phi^{2}}\right] \Psi=0
$$


Thus, substituting Eq. (70) into Eq. (69), we find that Eq. (71) should be written as

$$
\frac{d}{d r}\left(r^{2} \Delta \frac{d R}{d r}\right)+\left(\frac{\omega^{2} r^{2}}{c^{2} \Delta}-\lambda_{\operatorname{lm}}\right) R=0 .
$$

In order to obtain an equation which should be identified with one of the types of the Heun equation, let us introduce a new coordinate transformation corresponding to Eq. (72), given as follows:

$$
x=r^{2} \text {. }
$$

Thus, using this new coordinate, Eq. (73) turns into

$$
\Delta=1-\frac{r_{0}^{4}}{x^{2}} .
$$

Therefore, Eq. (75) is simply written as

$$
\Delta=\left(x-x_{+}\right)\left(x-x_{-}\right)=0
$$

whose solutions are the same given by Eqs. (76)-(77), namely,

$$
\begin{aligned}
& x_{+}=+x_{0}=+r_{0}^{2}, \\
& x_{-}=-x_{0}=-r_{0}^{2},
\end{aligned}
$$

and correspond to the new acoustic event horizons of the canonical acoustic black hole. Thus, Eq. (78) should read

$$
\begin{aligned}
& \frac{d^{2} R}{d x^{2}}+\left(\frac{1}{x-x_{+}}+\frac{1}{x-x_{-}}+\frac{3 / 2}{x}\right) \frac{d R}{d x} \\
& +\left[\frac{A_{1}}{x-x_{+}}+\frac{A_{2}}{x-x_{-}}+\frac{A_{3}}{x}+\frac{B_{1}^{2}}{\left(x-x_{+}\right)^{2}}+\frac{B_{2}^{2}}{\left(x-x_{-}\right)^{2}}+\frac{B_{3}^{2}}{x^{3}}\right] R=0 .
\end{aligned}
$$

From this point on, it is worth calling attention to the fact that the functional form of the radial part of the Klein-Gordon equation, for a massless scalar field in the spacetime of a canonical acoustic black hole, has changed. Then, we need to define some new coefficients, $A_{1}, A_{2}, A_{3}, B_{1}, B_{2}$, and $B_{3}$ which are given by

$$
\begin{aligned}
& A_{1}=\frac{-c^{2} \lambda\left(x_{+}^{2}+x_{-}^{2}\right)+2 c^{2} \lambda x_{+} x_{-}+\left(x_{-}-3 x_{+}\right) \omega^{2}}{4 c^{2} x_{+}^{2}\left(x_{+}-x_{-}\right)^{3}}, \\
& A_{2}=\frac{c^{2} \lambda\left(x_{+}^{2}+x_{-}^{2}\right)-2 c^{2} \lambda x_{+} x_{-}-\left(x_{+}-3 x_{-}\right) \omega^{2}}{4 c^{2} x_{-}^{2}\left(x_{+}-x_{-}\right)^{3}} \\
& A_{3}=\frac{-c^{2} \lambda\left(x_{+}+x_{-}\right)+\omega^{2}}{4 c^{2} x_{+}^{2} x_{-}^{2}}
\end{aligned}
$$




$$
\begin{aligned}
& B_{1}=\frac{\omega}{2 c \sqrt{x_{+}}\left(x_{+}-x_{-}\right)}, \\
& B_{2}=\frac{\omega}{2 c \sqrt{x_{-}}\left(x_{+}-x_{-}\right)}, \\
& B_{3}=\frac{i}{2} \sqrt{\frac{\lambda}{x_{+} x_{-}}} .
\end{aligned}
$$

Now, by setting the homographic substitution given by Eq. (79), Eq. (80) is now written as

$$
\begin{aligned}
& \frac{d^{2} R}{d z^{2}}+\left(\frac{1}{z}+\frac{1}{z-1}+\frac{3 / 2}{z-a}\right) \frac{d R}{d z} \\
& +\left[\frac{-A_{1} x_{+} / a}{z}+\frac{-A_{2} x_{+} / a}{z-1}+\frac{-A_{3} x_{+} / a}{z-a}\right. \\
& \left.+\frac{B_{1}^{2}}{z^{2}}+\frac{B_{2}^{2}}{(z-1)^{2}}+\frac{B_{3}^{2}}{(z-a)^{2}}\right] R=0,
\end{aligned}
$$

where the singular point $a$ is given by

$$
a=\frac{0-a_{1}}{a_{2}-a_{1}}=\frac{-x_{+}}{x_{-}-x_{+}} .
$$

Thus, the F-homotopic transformation of the dependent variable, $R(z) \mapsto U(z)$, given by Eq. (81), should read, in this case, as

$$
R(z)=z^{-\frac{1}{2}}(z-1)^{-\frac{1}{2}}(z-a)^{-\frac{3}{4}} U(z)
$$

Therefore, Eq. (84) is rewritten as

$$
\begin{aligned}
& \frac{d^{2} U}{d z^{2}}+\left\{\frac{\left(1+4 B_{1}^{2}\right) / 4}{z^{2}}+\frac{\left(1+4 B_{2}^{2}\right) / 4}{(z-1)^{2}}+\frac{\left(3+16 B_{3}^{2}\right) / 16}{(z-a)^{2}}\right. \\
& +\frac{\left(A_{1}+A_{3}\right) x_{+}+a\left[-2+\left(A_{1}+A_{2}\right) x_{+}\right]}{a} \frac{1}{(z-1)(z-a)} \\
& \left.+\frac{3+2 a-4 A_{1} x_{+}}{4} \frac{1}{z(z-1)(z-a)}\right\} R=0 .
\end{aligned}
$$

Now, let us consider the general Heun equation, whose canonical form is [59]

$$
\frac{d^{2} y}{d z^{2}}+\left(\frac{\gamma}{z}+\frac{\delta}{z-1}+\frac{\epsilon}{z-a}\right) \frac{d y}{d z}+\frac{\alpha \beta z-q}{z(z-1)(z-a)} y=0
$$


where $y(z)=\operatorname{HeunG}(a, q ; \alpha, \beta, \gamma, \delta ; z)$ is the general Heun function. This is a Fuchsian-type equation with regular singularities at $z=(0,1, a, \infty)$. The parameters $\alpha, \beta, \gamma, \delta, \epsilon, q, a$ are generally complex, arbitrary (except that $a \neq 0,1$ ), and related by

$$
\gamma+\delta+\epsilon=\alpha+\beta+1
$$

Its normal form is given by

$$
\begin{aligned}
& \frac{d^{2} U}{d z^{2}}+\left\{\frac{\left(2 \gamma-\gamma^{2}\right) / 4}{z^{2}}+\frac{\left(2 \delta-\delta^{2}\right) / 4}{(z-1)^{2}}+\frac{\left(2 \epsilon-\epsilon^{2}\right) / 4}{(z-a)^{2}}\right. \\
& +\frac{2 \alpha \beta-\delta \epsilon-\gamma(\delta+\epsilon)}{2} \frac{1}{(z-1)(z-a)} \\
& \left.+\frac{\gamma(a \delta+\epsilon)-2 q}{2} \frac{1}{z(z-1)(z-a)}\right\} R=0,
\end{aligned}
$$

where

$$
y(z)=z^{-\frac{\gamma}{2}}(z-1)^{-\frac{\delta}{2}}(z-a)^{-\frac{\epsilon}{2}} U(z) .
$$

Thus, the general solution of the radial part of the Klein-Gordon equation for a massless scalar particle in the spacetime of a canonical acoustic black hole, in the exterior region of the acoustic event horizon, has changed and hence Eq. (87) should read

$$
\begin{aligned}
R(z)= & z^{\frac{1}{2}(\gamma-1)}(z-1)^{\frac{1}{2}(\delta-1)}(z-a)^{\frac{1}{2}(\epsilon-2)}\left\{C_{1} \operatorname{HeunG}(a, q ; \alpha, \beta, \gamma, \delta ; z)\right. \\
& \left.+C_{2} z^{1-\gamma} \operatorname{HeunG}\left(a, q_{1} ; \alpha_{1}, \beta_{1}, \gamma_{1}, \delta ; z\right)\right\}
\end{aligned}
$$

where $C_{1}$ and $C_{2}$ are constants, and the parameters $\alpha, \beta, \gamma, \delta, \epsilon$, and $q$, which are given by Eqs. (88)-(92), are now given as follows:

$$
\begin{aligned}
\alpha= & \frac{1}{4 a}\left\{\sqrt{25 a^{2}-16 a^{2}\left(A_{1} x_{+}+A_{2} x_{+}+B_{1}^{2}+B_{2}^{2}+B_{3}^{2}\right)-16 a x_{+}\left(A_{1}+A_{3}\right)}\right. \\
& \left.+a\left[4 i\left(B_{1}+B_{2}\right)+4+\sqrt{1-16 B_{3}^{2}}\right]\right\} ; \\
\beta= & \frac{1}{2}\left[4 i\left(B_{1}+B_{2}\right)+\sqrt{1-16 B_{3}^{2}}+4\right]-\alpha ; \\
\gamma= & 1+2 i B_{1} \\
\delta= & 1+2 i B_{2} \\
\epsilon= & 1+\frac{1}{2} \sqrt{1-16 B_{3}^{2}} ; \\
q= & \frac{1}{4}\left\{2 i B_{1}\left[a\left(2+4 i B_{2}\right)+\sqrt{1-16 B_{3}^{2}}+2\right]+4 i a B_{2}+4 A_{1} x_{+}\right. \\
& \left.+\sqrt{1-16 B_{3}^{2}}-1\right\} .
\end{aligned}
$$


The parameters $\alpha_{1}, \beta_{1}, \gamma_{1}$, and $q_{1}$ are given by the relations

$$
\begin{aligned}
& \alpha_{1}=\alpha+1-\gamma, \\
& \beta_{1}=\beta+1-\gamma, \\
& \gamma_{1}=2-\gamma . \\
& q_{1}=q+(\alpha \delta+\epsilon)(1-\gamma) .
\end{aligned}
$$

Thus, the solution of the radial part of the Klein-Gordon equation has changed. It is no more given by a confluent Heun function, but now it is given by a general Heun function. As a consequence, the analogue Hawking radiation is modified. Then, in order to explicit this modification, we need to discuss the expansion of the general Heun function, as follows: If $\gamma \neq\{0,-1,-2, \ldots\}$, then from the Fuchs-Frobenius Theory, it follows that $\operatorname{HeunG}(a, q ; \alpha, \beta, \gamma, \delta ; z)$ exists, is analytic in the disk $|z|<1$, corresponds to exponent 0 at $z=0$ and assumes the value 1 there, and has the Maclaurin expansion

$$
\operatorname{HeunG}(a, q ; \alpha, \beta, \gamma, \delta ; z)=\sum_{j=0}^{\infty} b_{j} z^{j}
$$

where $b_{0}=1$, and

$$
\begin{aligned}
& a \gamma b_{1}-q b_{0}=0, \\
& X_{j} b_{j+1}-\left(Q_{j}+q\right) b_{j}+P_{j} b_{j-1}=0, \quad j \geq 1,
\end{aligned}
$$

with

$$
\begin{aligned}
P_{j} & =(j-1+\alpha)(j-1+\beta), \\
Q_{j} & =j[(j-1+\gamma)(1+a)+a \delta+\epsilon], \\
X_{j} & =a(j+1)(j+\gamma) .
\end{aligned}
$$

Thus, when $r \rightarrow r_{+} \Rightarrow x \rightarrow x_{+} \Rightarrow z \rightarrow 0$, Eq. (93) should have been

$$
R(r) \sim C_{1}\left(r-r_{+}\right)^{\frac{1}{2}(\gamma-1)}+C_{2}\left(r-r_{+}\right)^{-\frac{1}{2}(\gamma-1)} .
$$

Then, considering the time factor, near the canonical acoustic black hole event horizon $r_{0}$, Eq. (94) should be written as

$$
\Psi=\mathrm{e}^{-i \omega t}\left(r-r_{-}\right)^{ \pm \frac{1}{2}(\gamma-1)}
$$

and thus Eq. (95) should have be done by

$$
\frac{1}{2}(\gamma-1)=\frac{i}{2 \kappa_{0}} \omega
$$


where

$$
\kappa_{0}=2 c r_{0}^{3}
$$

From this point on, the analysis of the analogue Hawking radiation expressed by Eqs. (97)-(111) is all correct.

We apologize for the mistakes and express our gratitude to the colleagues for the comprehension.

Acknowledgements The authors would like to thank Conselho Nacional de Desenvolvimento Científico e Tecnológico $(\mathrm{CNPq})$ for partial financial support. H. S. V. is funded through the research Project No. 150640/2018-8. V. B. B. is partially supported through the research Project No. 305835/2016-5. The authors also would like to thank Prof. Luís C. B. Crispino for the fruitful discussions.

Publisher's Note Springer Nature remains neutral with regard to jurisdictional claims in published maps and institutional affiliations. 